\title{
Homogeneity in Control: Geometry and Applications ${ }^{\ddagger}$
}

\author{
Matthias Kawski ${ }^{1}$
}

\begin{abstract}
This tutorial presentation surveys some history and the geometric foundations for the use of homogeneity in the analysis and design of control systems, from classical applications to active research. Reflecting on the success of linear systems theory in a nonlinear world, homogeneous systems may be considered the next step, providing a richer class of models but still amenable to explicit analysis and design. Linearity means additivity together with homogeneity. But much of the effectiveness and power persists when additivity is lost: even without a superposition principle, relying only on homogeneity, stability is still determined by the dynamics on a reduced space that is a nonlinear analogue of the union of eigenspaces. Homogeneity immediately ties global to local properties.
\end{abstract}

The primary interest in homogeneous systems, as an intermediate step from linear to fully nonlinear systems, lies in their approximation properties. The following are almost true (and much research in the past decades has focused on the precise conditions when these are true): A system is controllable (feedback stabilizable) if and only if its homogeneous nilpotent approximation is controllable (respectively, stabilizable).

The classical definition of homogeneity, and many of its practical applications are firmly rooted in algebra. But much of its power in (controlled) dynamical systems stems from its geometric face, as a symmetry under a Lie group of continuous transformations. This naturally sets the stage for extending the use of homogeneity to infinite dimensional systems that include, in particular, dynamical systems with delays, and systems governed by partial differential equations, areas of very active current research, some of which will be featured in the other presentations of this tutorial session.

\section{INTRODUCTION}

This tutorial article on the role and use of homogeneity in control is ultimately driven by the problem of devising feedback schemes that stabilize a controlled dynamical system. Closely related to this central objective in control theory is the notion of controllability which serves as a preparatory step before stabilizability. The focus in this article is on finite dimensional, continuous-time, deterministic systems. But this presentation makes an effort to develop a unified framework that facilitates the extensions to e.g. infinite dimensional, discrete time, or stochastic systems.

The efforts to utilize homogeneity naturally aspire to follow the success of linear systems theory, which hinges on the class of linear systems being sufficiently rich to adequately model complex behaviors in a nonlinear world and at the same time possess a powerful algebraic toolbox for their analysis.

‡ This work was partially supported by the National Science Foundation through the grant DMS 09-08204

${ }^{1}$ Matthias Kawski is with the School of Mathematical and Statistical Sciences, Arizona State University, Tempe, AZ 85287-1804, USA. kawski@asu.edu
There is no hope for a similar complete analysis of general nonlinear systems, even only smooth ones. A natural intermediate step is the class of homogeneous systems. It contains the linear systems, and at the same time provides an infinitely richer class of models and still retains a large toolbox of analytical tools which is steadily improved by current research efforts. Moreover, homogeneous systems by their very nature naturally give rise to new paradigms such as finite-time stabilization [1], [2] that are not possible in the linear setting.

Homogeneous approximating systems have a similar relationship to full nonlinear models as do linear approximations in the sense that controllability and stabilizability properties naturally carry over. However, since the class of homogeneous systems is richer, they allow for closer approximations. The primary interest is in nonlinear systems whose linearization is neither controllable and stabilizable, but whose nonlinear controllability and stabilizability properties are captured by homogeneous approximating systems. Most elementary practical examples include everyday mechanical systems and objectives such as parallel parking a car (example 4).

The study of mathematical objects having homogeneity properties undoubtedly goes back millennia. More formal work in recent centuries ranges from, e.g. polynomials and algebraic geometry to operators and dynamical systems. A personal favorite and beautiful example for the use of homogeneity is in homotopy continuation methods for systems of polynomial equations, see the sequel of articles including [3]. Algebraically, the basic defining equation of homogeneity of a polynomial or function is the existence of an exponent $m$ such that for all constants $c$

$$
f(c x)=c^{m} f(x) \text { for all } x .
$$

The first thought may be real scalars $c$ and $x$. But such equation may as well interpreted as a relation among a pair of group actions on the domain and range of the function $f$. This article keeps an open eye for more general interpretations which extend the presented methods to new settings. Most of the time it is guided by the needs presented by the problem of local feedback stabilization of smooth nonlinear systems.

This setting has seen an explosive growth in activity since the late 1970s established Lie algebraic methods as the primary tool for the analysis of generally nonlinear systems of the form

$$
\dot{x}=f_{0}(x)+\sum_{i=1}^{m} u_{i} f_{i}(x) .
$$


where the state $x$ takes values in an $n$-dimensional smooth manifold, the $f_{i}$ are smooth vector fields on $M$, and the controls $u=\left(u_{1}, \ldots u_{m}\right)$ are measurable functions (defined on intervals $\left[0, T_{u}\right] \subseteq \mathbb{R}$ ) taking values in a (generally assumed to be) compact convex subset $U \subseteq \mathbb{R}^{m}$. Write $\mathcal{U}$ for the set of all such admissible controls, and $\xi(\cdot ; p, u)$ for the solution curve of system (2) with initial condition $x(0)=p$ corresponding to the control $u$.

Milestones are the main theorems [4], [5], [6] on (smalltime local) controllability of such systems that were established in the 1980s. Central to their proofs are constructions of homogeneous approximating systems which, on one hand, are amenable to direct analysis, and which, on the other hand, are sufficiently close approximations so that their controllability properties carry over to local controllability of the original system (2). Subsequently much work centered on the next step of designing feedback laws that stabilize such homogeneous systems with initial efforts including [7], [8], [9]. Mimicking the roadmap laid out by linear systems theory, the wishful chain of reasoning roughly followed these paradigms:

- If a system is controllable, then it has a controllable homogeneous approximation.

- If a homogeneous system is stabilizable, then it can be stabilized using a homogeneous feedback law.

- If a homogeneous system is controllable, then it is feedback stabilizable using a homogeneous feedback law.

- A homogeneous feedback law that stabilizes the homogeneous approximation also locally stabilizes the original system.

While none of the first three are correct, they nonetheless are very close to being true, and they provide a powerful strategy that applies to the vast majority of systems of practical interest. The careful analysis of the gaps that cause these implications to fail continues to be an area of active research. The system presented in [10] demonstrates that failure of the first implication occurs (only) in systems that are of much higher order than commonly encountered. An illuminating counterexample of the second implication presented in [11], see also [12], in some sense still validates the general strategy of first trying homogeneous stabilizing feedback which has been successfully employed in many settings, e.g. [2], [7], [13], [14], [15], [16], including generalizations to adaptive and uncertain systems, systems with delays and others. Due to well-known topological obstacles (degree arguments) [17], [18], [19] it is clear that in general no continuous stabilizing feedback laws may exist even for controllable systems. However, using a simple periodically timevarying modification of state-feedback laws it was shown that controllability implies stabilizability in this enhanced sense [19]. Substantial interest remains in identifying the precise topological mechanisms that are responsible for a lack of continuous feedback stabilizability of certain homogeneous systems due to resonances of certain system parameters [9], [11], [20]. Finally, the last implication was shown to be true and is based on the existence of homogeneous Lyapunov functions. The critical contribution made in [21] establishes the existence of smooth homogeneous Lyapunov functions even if the system is not locally Lipschitz continuous. This is needed as already [8] demonstrated that nonlinearly controllable systems whose linear approximation fails to capture the controllability necessitate nondifferentiable (but still continuous) feedback laws for stabilization.

The subsequent sections of this tutorial are organized as follows. First we give a quick review of controllability and nilpotent homogeneous approximating systems and their properties, as these establish the need for a specific notion of homogeneity. Then we focus on the construction of homogeneous stabilizing feedback laws and limitations of this quest. This is followed by a more geometric view which sets the stage for extending the paradigms and approaches to more diverse settings.

\section{CONTROLLABILITY AND HOMOGENEITY}

This section surveys classical controllability results for systems of the form (2) with special focus on nilpotent approximating systems. Their distinguishing role as approximating systems justifies that such systems with their special structure deserve special attention and study. It is their natural graded structure that gives rise to a specific associated notion of homogeneity employed in the sequel.

The graded structure arises from expanding the solution curve $\xi(\cdot ; p, u)$ as an exponential Lie series. More specifically, identify the vector field $f=\left(f_{1}, \ldots f_{n}\right)^{T}$ with the first order partial differential operator $f=\sum_{i=1}^{n} f_{i} \frac{\partial}{\partial x_{i}}$. Throughout assume that the fields $f_{j}$ are analytic. The ChenFliess series

$$
\varphi(\xi(t ; p, u))=\sum_{w \in Z} \Upsilon_{w}(t, u)\left(f_{w} \varphi\right)(p)
$$

is an asymptotic series for the evolution of an analytic output function $\varphi: M \mapsto \mathbb{R}$ along the solution curves of system (2). Here $Z$ is the set of all words (or finite sequences) $\left(i_{1}, i_{2}, \ldots i_{s}\right) \in\{1,2, \ldots, n\}^{s}, s \in \mathbb{Z}_{0}^{+}, f_{\emptyset} \varphi=\varphi$, and inductively $f_{a w}=f_{a}\left(f_{w} \varphi\right)$ is a higher order Lie derivative of $\varphi$ along the system vector fields. The coefficients $\Upsilon_{w}$ are iterated integrals of the control inputs with $\Upsilon_{\emptyset}=1$ and inductively $\Upsilon_{a w}(t, u)=\int_{0}^{t} \Upsilon_{w}(s, u) \cdot u_{a}(s) d s$. This series may be understood as a noncommutative generalization of the Taylor (or Volterra) expansion of the solution of an uncontrolled dynamical system. There are many ways to rewrite this series in more useful formats, e.g. as a directed exponential product [22], or as the exponential of a Lie series, as a continuous analogue of the Baker-CampbellHausdorff formula [23]. In the end, what matters in the context of this article is that the homogeneous systems considered in the sequel naturally arise as leading terms in suitable truncations of such series or products. With stabilization the final objective, an intermediate step is controllability. In this setting the natural notion is:

Definition 2.1: The system (2) is called small-time locally controllable (STLC) about an equilibrium point $x_{0} \in M$ 
of $f_{0}$ if for every $T>0$ the reachable set $\mathcal{R}_{T}\left(x_{0}\right)=$ $\left\{\xi\left(T ; x_{0}, u\right): u \in \mathcal{U}\right\}$ contains $x_{0}$ in its interior.

Well-known sufficient conditions for STLC involve Lie ranks and linear relationships between iterated Lie brackets of the system vector fields $f_{i}$. More specifically, for smooth vector fields $u, v$ write $(\operatorname{ad} v, w)=[v, w]=v \circ w-w \circ v$ for their commutator (which is again a vector field, i.e. first order partial differential operator), and inductively $\left(\operatorname{ad}^{k+1} v, w\right)=$ $\left[v,\left(\operatorname{ad}^{k} v, w\right)\right]$. Write $L(\mathcal{F})$ for the Lie algebra generated by the collection $\mathcal{F}=\left\{f_{0}, f_{1}, \ldots f_{m}\right\}$ of vector fields, and for any multi-index $I=\left(i_{0}, i_{1}, \ldots i_{m}\right)$ with $i_{j} \in \mathbb{Z}_{0}^{+}$write $L^{I}(\mathcal{F})$ for the linear span of all iterated Lie brackets that involve $i_{j}$ factors of the vector field $f_{j}$.

Sufficient conditions for STLC about an equilibrium point $x_{0}$ of $f_{0}$ are given in [5], [6]. The system [2] is STLC about $p$ if $L(\mathcal{F})(p)=T_{p} M$ spans the tangent space at $p$, and there exist a weight $\theta \in[0,1]$ such that whenever $\ell_{0}$ is odd, and $\ell_{1}, \ldots \ell_{m}$ are even nonnegative integers then $L^{\ell}(\mathcal{F})(p) \subseteq$ $\sum_{\ell^{\prime}} L^{\ell^{\prime}}(\mathcal{F})(p)$ where the sum is taken over all multi-indices $\ell^{\prime}$ such that $\theta \ell_{0}^{\prime}+\sum_{j=1}^{m} \ell_{j}^{\prime}<\theta \ell_{0}+\sum_{j=1}^{m} \ell_{j}$. The special case when $\theta=0$ and $m=1$ is known as the Hermes condition [5]. A critical strategy in the original proof uses nilpotent approximating systems whose homogeneity properties make their controllability properties amenable to explicit analysis. In particular, systems that satisfy this general sufficient condition for STLC have homogeneous, nilpotent, controllable, approximating systems. The following example illustrates how the Lie algebraic conditions come into play, how they lead to a natural notion of homogeneity, and homogeneous approximating systems. Due to their special role these deserve special attention and detailed analysis. The algorithmic procedure presented here, and the subsequent stability arguments follow the presentation in [24].

Consider the example of parallel parking a car (or, rather, a unicycle). The states are the location and speed of the car in the plane, the angle it makes with respect to a fixed direction, and the angle of the front wheel and the car. Natural controls are the forward acceleration and angular velocity of the steering wheel. The model is

$$
\begin{aligned}
& \dot{x}_{1}=x_{3} \cos x_{4} \\
& \dot{x}_{2}=x_{3} \sin x_{3} \\
& \dot{x}_{3}=u_{1} \\
& \dot{x}_{4}=x_{3} \sin x_{5} \\
& \dot{x}_{5}=u_{2}
\end{aligned}
$$

is in the form of the system (2) with the drift vector field $f_{0}=x_{3} \cos x_{4} \frac{\partial}{\partial x_{1}}+x_{3} \sin x_{4} \frac{\partial}{\partial x_{2}}+x_{3} \sin x_{5} \frac{\partial}{\partial x_{4}}$, and the controlled fields $f_{1}=\frac{\partial}{\partial x_{3}}$ and $f_{2}=\frac{\partial}{\partial x_{5}}$.

The algorithm needs to keep track of the internal structure of the Lie brackets employed, and consequently one needs to consider formal brackets in a free Lie algebra generated by $(m+1)$ letters, or indeterminates $X_{0}, X_{1}, \ldots X_{m}$. This is needed as e.g. the vector fields $f_{1}$ and $\left[f_{1}, f_{2}\right]$ may be equal, yet one needs to keep track of the length of the iterated Lie brackets employed. The obvious substitution homomorphism maps the formal brackets to analytic vector fields, and in the sequel is denoted by simply replacing $X$ by $f$ in any expressions.

Recursively calculating the iterated Lie brackets of these vector fields leads to the choice of the following formal brackets $X_{\pi_{1}}=X_{1}, X_{\pi_{2}}=X_{2}, X_{\pi_{3}}=\left[X_{1}, X_{0}\right]$, $X_{\pi_{4}}=\left[X_{1},\left[X_{2}, X_{0}\right]\right], X_{\pi_{5}}=\left[\left[X_{1},\left[X_{2}, X_{0}\right]\right],\left[X_{1}, X_{2}\right]\right]$ whose images $f_{\pi_{j}}(p)$ span the tangent space at $p=0$. In particular, this establishes that this system is STLC about $p$ by virtue of the general sufficient condition [6].

Define the weight of a formal bracket as the sum of the weights of its factors, i.e. the weight is a semi-group homomorphism from the set of iterated formal brackets to the positive integers, and thus endows the first set with a natural graded structure. Customarily, corresponding to the general sufficient condition for STLC, one chooses $w_{i}=1$ for $j \geq 1$ and any value $w_{0} \in[0,1]$. Using the weights $w_{0}=w_{1}=w_{2}=1$ for the generators $X_{0}, X_{1}$ and $X_{2}$, these $X_{\pi_{j}}$ are formal brackets of minimal weight such that the corresponding iterated Lie brackets $f_{\pi_{j}}$ of vector fields span the tangent space at $p=0$.

Corresponding to the specific choice of weights $w_{j}$ of the generators $X_{0}, X_{1}, \ldots X_{m}$ and choice of the distinguished formal brackets $X_{\pi_{j}}$ whose images $f_{\pi_{j}}(p)$ space the tangent space at $p$, define positive dilation exponents $r_{1}, \ldots r_{m}$ as the weights of the formal brackets $X_{\pi_{j}}$. In this example these agree with the lengths $r=(1,1,2,3,5)$ of these formal brackets. These are the weights that define the notion of homogeneity employed later.

To proceed with the construction of a nilpotent approximating system, after the choice of such suitable weights $w_{j}$ and formal iterated brackets $X_{\pi_{j}}$, one generally first performs a constant linear coordinate change $y=Q x$ such that in the new coordinates the corresponding vector fields $f_{\pi_{j}}$ for $j \geq 1$ agree at the point $p$ with the coordinate directions $f_{\pi_{j}}(p)=\left.\frac{\partial}{\partial y_{j}}\right|_{p}$. In this example such change is just permutation of the coordinates.

Next, expand each of the components of the vector fields $f_{j}$ into Taylor series about $p$ in the new coordinates $y_{i}$. Assigning to each monomial $m(x)=c y_{1}^{s_{1}} y_{2}^{s_{2}} \ldots y_{n}^{s_{n}}$ the weight $w_{m}=r_{0} m_{0}+r_{1} m_{1}+\ldots r_{n} m_{n}$, truncate in each component $\left\langle d y_{i}, f_{j}\right\rangle$ the Taylor series at terms of weight $w_{i j}=r_{j}-w_{i}$. Denoting the resulting vector fields by $g_{j}$, one obtains in this example $g_{0}=y_{1} \frac{\partial}{\partial y_{3}}+y_{1} y_{2} \frac{\partial}{\partial y_{4}}+y_{1} y_{4} \frac{\partial}{\partial y_{5}}$, $g_{1}=\frac{\partial}{\partial y_{1}}$, and $g_{2}=\frac{\partial}{\partial y_{2}}$. By construction, compare the subsequent sections, the Lie algebra generated by these vector fields is nilpotent (see below), and in particular finite dimensional. Consequently, solutions may be computed using simple quadratures, and e.g. the exponential product expansion of the Chen-Fliess series for this systems is a finite product. By construction, this system is again STLC, and its solution curves closely approximate in a well defined sense those of the original system (4). The next section shows how corresponding feedback stabilization schemes designed for such approximating system (locally) achieve the desired objective for the original system

Written as a system of differential equations this approx- 
imate model takes a simple polynomial cascade form

$$
\begin{aligned}
& \dot{y}_{1}=u_{1} \\
& \dot{y}_{2}=u_{2} \\
& \dot{y}_{3}=y_{1} \\
& \dot{y}_{4}=y_{1} y_{2} \\
& \dot{y}_{5}=y_{1} y_{4} .
\end{aligned}
$$

In general, the algorithm presented above alone need not necessarily yield a homogeneous system, but after another simple triangular polynomial coordinate change (with inverse of the same form), an adapted chart achieves the desired homogeneous format, compare [4], [24], [25], [26]. This homogeneity takes the form that the polynomial in the $i$-th component of the vector field $g_{j}$ has weight $r_{i}-w_{j}$. Recalling $r=(1,1,2,3,5)$, and $y_{1}$ has weight $r_{1}=1=r_{3}-1, y_{1} y_{2}$ has weight $r_{1}+r_{2}=2=r_{4}-1$, and $y_{1} y_{4}$ has weight $r_{1}+r_{2}=4=r_{5}-1$.

\section{HOMOGENEITY AND STABILIZATION}

The graded structure of the nilpotent approximating systems discussed in the previous section is closely related to similar approximations utilized for second order hypoelliptic differential operators, compare [27], [28]. This structure gives rise to a natural algebraic (and coordinate dependent) notion of weighted homogeneity on Euclidean space. (Such weighted notions of homogeneity have been utilized in various places before.)

Definition 3.1: Suppose $1 \leq r_{1} \leq \ldots \leq r_{n}$ is a finite nondecreasing sequence of real numbers. Then $\Delta=\left\{\Delta_{t}\right\}_{t>0}$ is a group of dilations acting on $\mathbb{R}^{n}$ defined by:

$$
\Delta_{t}(x)=\left(t^{r_{1}} x_{1}, t^{r_{2}} x_{2}, \ldots t^{r_{n}} x_{n}\right)
$$

A function $\varphi: \mathbb{R}^{n} \mapsto \mathbb{R}$ is called homogeneous of degree $m$ with respect to the group $\Delta$ if $\varphi \Delta_{t}=t^{m} \cdot \varphi$. The set of functions that are homogeneous of degree $m$ is denoted by $H_{m}$.

A vector field $f: \mathbb{R}^{n} \mapsto T \mathbb{R}^{n}$ is homogeneous of degree $k$ is for every $m$ and every $\varphi \in H_{m}^{1}=C^{1}\left(\mathbb{R}^{n}, \mathbb{R}\right) \cap H_{m}$, $f \varphi \in H_{m+k}$. The set of all vector fields homogeneous of degree $k$ is denoted by $\underline{n}_{k}$, and write $\underline{n}_{k}^{1}$ for the subset of continuously differentiable vector fields.

The choice of the standard dilation $r=(1,1 \ldots, 1)$ recovers the classical notion of homogeneity.

Note that a vector field $f=\sum_{j=1}^{n} f_{j} \frac{\partial}{\partial x_{j}}$ is homogeneous of degree $k$ if and only if each component $f_{i}$ is homogeneous of degree $\left(k+r_{i}\right)$. Thus, in particular, call a linear vector field (as in a linear system $\dot{x}=A x+B u$ ) homogeneous of degree zero with respect to the standard dilation.

It is evident that the homogeneous sets $H_{m}$ and $\underline{n}_{k}$ are linear spaces for every $m$ and every $k$. Moreover, they provide natural graded structures on suitable subspaces of functions and vector fields.

Proposition 3.1: For all $m, m^{\prime}, k, k^{\prime}$,

$$
\begin{aligned}
H_{m} \cdot H_{m^{\prime}} & \subseteq H_{m+m^{\prime}} \\
\underline{n}_{k} H_{m}^{1} & \subseteq H_{m+k}, \text { and } \\
{\left[\underline{n}_{k}^{1}, \underline{n}_{k^{\prime}}^{1}\right] } & \subseteq \underline{n}_{k+k^{\prime}} .
\end{aligned}
$$

In most applications in this setting, and for most of the sequel we may restrict ourselves to $r_{i}$ being positive integers. Moreover, whereas the original data may be analytic, stabilizing feedback laws often are only continuous, and not continuously differentiable in which case one needs to keep track of degrees of smoothness in relations such as the one in the preceding proposition. This tutorial minimizes distracting notation for this, but cannot do entirely without.

Note that the Lie algebra generated by any collection of vector fields $f_{j} \in \bigoplus_{k<0} \underline{n}_{k}$ is necessarily nilpotent since $\underline{n}_{k}=\{0\}$ for all $k<-\max _{j=1}^{n} r_{j}$.

It is convenient to also introduce a gauge, call it a homogeneous norm, $\|\cdot\|: \mathbb{R}^{n} \mapsto \mathbb{R}_{0}^{+}$by (for some $p>0$ )

$$
\|x\|_{p}=\left(\sum_{j=1}^{n}\left|x_{j}\right|^{p / r_{j}}\right)^{p} .
$$

It is immediate that $\|\cdot\|_{p} \in H_{1}$. If all $r_{j}$ are positive integers, then the most common choice a positive integer $p$ that is a multiple of $2 r_{j}$ for all $j \leq m$, and in this case $\|\cdot\|_{p} \in H_{1}^{1}$. Define the homogeneous (unit-)sphere as $S_{\Delta}=\|\cdot\|_{p}^{-1}(1)=$ $\left\{x \in \mathbb{R}^{n}:\|x\|=1\right\}$, and the projection $\pi: \mathbb{R}^{n} \backslash\{0\} \mapsto S_{\Delta}$ along the rays $\Delta_{t}(\cdot)$

A key property of every homogeneous system is that its dynamics can be decomposed into a radial dynamics, and dynamics on the compact sphere. As further discussed in the next section, it is the compactness of the space underlying the latter dynamics that gives rise to novel stabilization algorithms that focus on eigenray placement, rather then the pole placement (or eigenvalue placement) that is at the heart of linear system theory.

Next fix precise terminology for notions of stability. Consider dynamical systems $\dot{x}=F(x)$ on a smooth manifold $M$ defined by continuous vector fields $F: M \mapsto T M$ that have forward unique solutions to initial value problems. The local flows are denoted by $\Phi: \Omega \subseteq \mathbb{R} \times M \mapsto M$ and satisfy $\frac{d}{d t} \Phi(t, x)=F(\Phi(t, x))$. Where suitable, write $\Phi_{t}$ for $\Phi(t, \cdot)$.

Definition 3.2: An equilibrium point $x_{0} \in M$, i.e. $F\left(x_{0}\right)=0$ is called stable if for every open neighborhood $V$ of $x_{0}$ there exists an open neighborhood $U$ of $x_{0}$ such that for every $t \geq 0, \Phi(t, U) \subseteq V$.

An equilibrium point $x_{0}$ is locally attractive if there exists an open neighborhood $V$ of $x_{0}$ such that for every $x \in V$ and every open neighborhood $U$ of $x_{0}$ there exists a $T \geq 0$ such that for all $t \geq T, \Phi(t, x) \subseteq U$.

An equilibrium point $x_{0}$ is (locally) asymptotically stable if it is stable and (locally) attractive.

An equilibrium point $x_{0}$ is (locally) exponentially stable if there exist constants $C, \alpha>0$ such that for all $x \in \mathbb{R}^{n}$ 
(respectively, for all $x$ in some open neighborhood of $x_{0}$ ) for all $t \geq 0,\left\|\Phi(t, x)-x_{0}\right\| \leq C\left\|x_{0}\right\| e^{-\alpha t}$ (where $\|\cdot\|$ is any norm in $\mathbb{R}^{n}$ ).

The notion of exponential stability specializes to homogeneous systems, using homogeneous norms (assuming $x_{0}=$ $0)$.

Following are a few comments about linear systems, which serve as a model for investigations of, and constructions for, homogeneous systems. For linear systems $\dot{x}=A x+B u$ with $A$ and $B n \times n$ and $n \times m$ matrices, respectively, the Kalman rank condition determines controllability. Due to their homogeneity local and global controllability agree (assuming that the set $U$ is taken as $U=\mathbb{R}^{m}$, i.e., is unbounded), and are equivalent to the rank of the compound matrix $\left[B, A B, A^{2} B, \ldots, A^{n-1} B\right]$ being $n$. By performing a linear coordinate change that brings the pair $(A, B)$ into controller form, basically corresponding to companion matrices, it is routine to see that via linear feedback $u=K x$ determined by a constant $m \times n$ matrix $K$ one can arbitrarily assign the eigenvalues of the matrix $(A+B K)$, and thus render the closed loop system $\dot{x}=(A+B K) x$ globally exponentially stable.

A general nonlinear system of form (2) with linearization given by $A=\left(D f_{0}\right)\left(x_{0}\right)$ and $B=\left[f_{1}\left(x_{0}\right), \ldots f_{m}\left(x_{0}\right)\right]$ about an equilibrium point $x_{0}$ of $f_{0}$ maybe considered a perturbation of $\dot{x}=A x+B u$. As a consequence of Lyapunov's first (or indirect) method it is rendered locally asymptotically stable by the same feedback law $u=K x$. This may be proved directly by estimating the terms in the variation of parameters formula. Alternatively, one may argue using quadratic Lyapunov functions, which then generalize to homogeneous Lyapunov functions. In particular, if all eigenvalues of $(A+B K)$ have negative real part, then there exist positive definite matrices $P$ and $Q$ such that the derivative of $V(x)=x^{T} P x$ along trajectories of $\dot{x}=$ $A x+B u$ is $\dot{V}(x)=-x^{T} Q x$. Consequently, after adding the higher order perturbations $\left(f_{0}(x)-\left(D f_{0}\right)\left(x_{0}\right)\left(x-x_{0}\right)\right)$ and $\left(f_{i}(x)-f_{j}\left(x_{0}\right)\right) u_{j}$ the time derivative of the same $V(x)=x^{T} P x$ is still $\dot{V}(x)<0$ for all $x \neq x_{0}$ in some deleted open neighborhood of $x_{0}$. The assertion follows from Lyapunov's second (or direct) method.

In principle, this fundamental and well-known strategy carries over from the linear to the homogeneous case. The first steps do not work for all systems and are discussed below, but the final Lyapunov argument is immediate, see e.g. [24], [29] and a simple form (for integer exponents $r_{j}$ ) is restated here.

Proposition 3.2: Suppose $F, F_{0}: \mathbb{R}^{n} \mapsto T \mathbb{R}^{n}$ are complete continuous vector fields such that $F_{0} \in \underline{n}_{\alpha}$ is homogeneous with respect to a dilation $\Delta$ defined by integer exponents $1 \leq r_{j} \leq \ldots r_{n}$, and $V \in H_{\beta}^{1}$ is a smooth homogeneous function such that $V(0)=0$ and $V(x)>0$ and $\dot{V}(x)=\left(F_{0} V\right)(x)<0$ for all $x \neq x_{0}$. If there exist $C>0, \gamma>\alpha$, and an open neighborhood $U$ of 0 such that for all $x \in U,\left\|\left(F-F_{0}\right)(x)\right\|<C\|x\|^{\gamma}$ then $\dot{V}(x)=$
$(F V)(x)<0$ for all $x$ in some deleted neighborhood $U_{1}$ of 0 .

The norms in this statement are the homogeneous norms with respect to the dilation $\Delta$. In practice, one most commonly uses this argument when $\left(F-F_{0}\right)$ is written as a converging series of vector fields $F_{j}$ each of which is homogeneous of order strictly higher than $F_{0}$. The field $F$ itself arises as the closed loop vector field $f_{0}(x)+\sum k_{i}(x) f_{j}(x)$ for a continuous feedback law $u=k(x)$.

As a simple example consider a general planar analytic system of form (2) that is STLC about $x_{0}=0$. By virtue of the Hermes condition (as a special case of [5], [6], and complementary necessary condition) for STLC there exist some integer $r \geq 0$ such that $\left(\operatorname{ad}^{j} f_{1}, f_{0}\right)(0) \wedge f_{1}(0)=0$ for all $j \leq 2 \ell$ and $\left(\operatorname{ad}^{2 \ell+1} f_{1}, f_{0}\right)(0) \wedge f_{1}(0) \neq 0$ (where $\wedge$ is the exterior product). Consider the special case of $\ell=1$, as the general case is completely analogous, only requiring more burdensome notation. After a suitable choice of local coordinates, and rescaling of time, the nilpotent approximating system can be written for $\sigma \in\{-1,0,1\}$ as

$$
\begin{aligned}
\dot{x} & =u \\
\dot{y} & =\sigma y-x^{3} .
\end{aligned}
$$

which was analyzed in [8]. This system is homogeneous of degree 3 with respect to the group of dilations defined by the exponents $r=(1,3)$. Of particular interest is the case of $\sigma=1$. In this case no continuously differentiable feedback law $u=h(x)$ can possibly stabilize the system as the linearization of the resulting closed loop system necessarily would have the positive eigenvalue +1 . The Ansatz

$$
V=-\int f_{2}(x, y) d x+C(y)=\frac{1}{4} x^{4}-x y+C(y)
$$

for a (control) Lyapunov function (or Hamiltonian) $V$ suggests to choose $C(y)=e y^{4 / 3}$ and

$$
u=h_{0}(x, y)=\frac{\partial V}{\partial y}=-x-\frac{4 e}{3} y^{1 / 3} \in H_{1} .
$$

The choice of the exponent $\frac{4}{3}$ assures that the Lyapunov function $V$ and its derivative $\dot{V}$ in the direction of the closed loop system $\left(f_{0}+h_{0} f_{1}\right) \in \underline{n}_{0}$ are homogeneous with respect to the dilation, i.e. $V, \dot{V} \in H_{4}$. By construction the feedback law $h_{0}$, and thus the vector field $\left(f_{0}+h_{0} f_{1}\right)$ are not locally Lipschitz continuous, but only Hölder continuous. Thus the basic existence theorems for unique solutions of initial value problems do not apply. Yet, as is demonstrated in [8] this system (and systems constructed along the same lines) have unique solutions to initial value problems, and generalized Lyapunov arguments still apply. By construction $V$ is constant along trajectories of the closed loop system with feedback law $u=h_{0}(x, y)$. Compactness of the sublevel sets $V^{-1}([0, C]), C>0$ is guaranteed if $e>$ $\frac{3}{4}$, and hence the closed loop system is Lyapunov stable. Choosing $u=h(x)=-a x+b y^{1 / 3}$ with $b>a>1$ is easily shown (using Lasalle's invariance principle) to render the system globally asymptotically stable [8]. By virtue of 
the proposition 3.2 one concludes that every small-time locally controllable planar analytic system of form (2) is continuously feedback stabilizable [30].

This example naturally lays out a possible strategy for stabilizing general, nonlinearly controllable systems. Of course, already in dimension three, even for simple polynomial homogeneous systems the explicit construction of a homogeneous (control) Lyapunov function is highly nontrivial. Concerted efforts by many researchers, e.g. [7], [9], [13], [11] to name only a few, have managed to perform similar constructions for various classes of such systems, but there remain open questions about topological obstacles to the existence of such continuous stabilizing feedback laws which typically take the form of resonances between the system parameters which may make continuous stabilization impossible [31], [9], [20], [11]. A typical such example is the system

$$
\begin{aligned}
\dot{x} & =u \\
\dot{y} & =\lambda y-x^{3} . \\
\dot{z} & =\mu z-x^{9} .
\end{aligned}
$$

which exhibits a resonance at $\mu=3 \lambda>0$ which presents a topological obstacle to feedback stabilizability by a continuous homogeneous feedback law [9]. It was shown in [11] that some homogeneous systems are stabilizable by continuous feedback laws, but not by homogeneous continuous feedback laws. However, this does not take away the above approach which owes much of its attractiveness to the use of homogeneous Lyapunov functions and their use for perturbed systems as in proposition 3.2 In general it may be very challenging to explicitly construct such homogeneous (control) Lyapunov functions, but a critical result by Rosier [21] assures that such homogeneous Lyapunov functions always exist under very mild hypotheses. As is clear from the construction above, it is of critical importance that Rosier's theorem does not require the homogeneous vector field to be Lipschitz continuous, but still yields a homogeneous and smooth Lyapunov function.

\section{GEOMETRY: LIE SYMMETRY}

The preceding sections demonstrated how (a suitable notion of weighted) homogeneity is a powerful tool for analyzing nonlinear controllability, system approximation and characterizing tolerable perturbations, and ultimately, feedback stabilization. This section addresses, and removes, an awkward feature of the definition 3.1 of homogeneity which critically depends on a choice of local coordinates: After all, stability and stabilizability are geometric, topological, and not linear algebra, nor even metric, concepts.

Indeed, that special notion of homogeneity directly arose from the specific graded and filtered structure of the Lie algebra generated by the system vector fields of (2), and consequent approximations based on the Chen Fliess series (3). This section removes this awkward use of local coordinates and demonstrate that a purely geometric notion of homogeneity captures the fundamental properties needed in stability and stabilizability arguments. This generalization does not provide any significant practical improvement for systems such as (2) and those discussed previously (as they are already well served by the special, coordinate based definition). But this geometric notion not only lays bare what really makes homogeneity so useful for stability and stabilization, but it also opens the door to generalizations far beyond finite dimensional affine systems such as (2). Some of these are pointed out briefly in the next section, including systems with delays and other infinite dimensional systems, in particular, systems governed by partial differential equations.

This tutorial presentation loosely follows [32] to which the interested reader is referred to for more technical details, see also [1] for a related treatment. The natural starting point is a generalization of the group action (6). In most generality this need not be smooth, but for the sake of clarity, consider here the more tangible setting where the group action has a smooth infinitesimal generator. This approach is inspired by the classical characterization (commonly termed Euler's formula) of a smooth function $\varphi: \mathbb{R}^{n} \mapsto \mathbb{R}$ being homogeneous of degree $m$ if and only if

$$
\nu \varphi=m \varphi
$$

where $\nu: \mathbb{R}^{n} \mapsto T \mathbb{R}^{n}$ is the vector field defined by

$$
\nu(x)=\sum_{j=1}^{n} r_{j} x_{j} \frac{\partial}{\partial x_{j}}
$$

which is now commonly called Euler vector field [31]. The most familiar notion corresponds to all $r_{j}=1$, and the notion of weighted homogeneity employed above allows each $r_{i}$ to be a positive real numbers (or integers). However, for most of the stability and stabilizability properties and results, this specific form of the generator $\nu$ is not at all needed. Indeed, all that is needed (in finite dimensions) is the following:

Definition 4.1: A geometric group dilations is generated by a continuously differentiable vector field $\nu$ on $\mathbb{R}^{n}$ that is complete (i.e., its integral curves are defined for all $t \in(-\infty, \infty))$ and such that the origin is an asymptotically stable equilibrium of $-\nu$ (i.e., of the system of differential equations $\dot{x}=-\nu(x)$.

In the sequel $\nu$ always refers to such a vector field. This section rephrases the key notions and properties of homogeneity and argue why and how most tools and theorems generalize to this setting. This is not to be seen as a final abstraction, but as the starting point to extend this approach using homogeneity beyond finite dimensional continuoustime deterministic systems that are affine in the control.

Given such a vector field $\nu$ define the associated group actions (in both additive and multiplicative notation) in terms of the flow of $\nu$. Write $\Phi: \mathbb{R} \times \mathbb{R}^{n} \mapsto \mathbb{R}^{n}$ for the flow of $\nu$, i.e., for all $(t, x) \in \mathbb{R} \times \mathbb{R}^{n}, \Phi(0, x)=x$ and $\frac{d}{d t} \Phi(t, x)=$ $\nu(\Phi(t, x))$. To connect with the multiplicative notion of a group of dilation as presented above, define the associated 
maps $\Delta: \mathbb{R}^{+} \times \mathbb{R}^{n} \mapsto \mathbb{R}^{n}$ by $\Delta_{s}(x)=\Phi(\log s, x)$. This ensures the desired group properties for all $s, s^{\prime} \in \mathbb{R}^{+}$, all $t, t^{\prime} \in \mathbb{R}$, and all $x \in \mathbb{R}^{n}$

$$
\Delta_{s} \circ \Delta_{s^{\prime}}=\Delta_{s s^{\prime}} \quad \text { and } \Phi\left(t, \Phi\left(t^{\prime}, x\right)\right)=\Phi\left(t+t^{\prime}, x\right) .
$$

The group actions of $\Phi$ (and $\Delta$ ) on the state space $\mathbb{R}^{n}$ immediately induce corresponding group actions of the spaces of (output) functions, vector fields, and endomorphisms of $\mathbb{R}^{n}$. While in general none of these need to be smooth, this tutorial considers the special case where they are smooth, and thus their homogeneity may also be characterized in terms of the field $\nu$, and the graded structure of the Lie algebra of homogeneous vector fields may be stated in terms of Lie brackets, compare [32] for further details and elementary proofs.

Proposition 4.1: Given a geometric group of dilations $\Phi$ (or $\Delta$ ) generated by a vector field $\nu$ on $\mathbb{R}^{n}$ as above, and $m \in \mathbb{R}$, and a smooth function $\varphi: \mathbb{R}^{n} \mapsto \mathbb{R}$ the following are equivalent

- $\nu \varphi=m \varphi$,

- $\varphi \circ \Delta_{s}=s^{m} \cdot \varphi$ for all $t \in \mathbb{R}$, and

- $\varphi \circ \Phi_{t}=e^{m t} \cdot \varphi$ for all $s>0$.

Any function $\varphi$ that satisfies these is called homogeneous of degree $m$, written $\varphi \in H_{m}^{1}$.

Proposition 4.2: For $\nu, \Phi$ and $\Delta$ as above, $k \in \mathbb{R}$, and a smooth vector field $f$ with associated flow $\Psi$, the first three statements are equivalent, and they imply the fourth:

- $[\nu, f]=k f$,

- $\Psi_{\tau} \circ \Phi_{t}=\Phi_{t} \circ \Psi_{e^{k t} \tau}$ for all $t, \tau \in \mathbb{R}$

- $\Psi_{\tau} \circ \Delta_{s}=\Delta_{s} \circ \Psi_{s^{k} \tau}$, for all $\tau \in \mathbb{R}$ and $s>0$, and

- $f H_{m}^{1} \subseteq H_{m+k}$.

Any vector field $f$ that satisfies these is called homogeneous of degree $k$, written $f \in \underline{n}_{k}^{1}$.

Proposition 4.3: For $\nu, \Phi$ and $\Delta$ as above, $k, \ell \in \mathbb{R}$, and a smooth map $\Psi: \mathbb{R}^{n} \mapsto \mathbb{R}^{n}$ then the second and third of the following statements are equivalent, and imply the first:

- $\varphi \circ \Psi \in H_{m+\ell}$ for all $m$ and all $\varphi \in H_{m}$,

- $\Psi \circ \Phi_{t}=\Phi_{\ell t} \circ \Psi$ for all $t \in \mathbb{R}$

- $\Psi \circ \Delta_{s}=\Delta_{s^{\ell}} \circ \Psi$, for all $s>0$, and

- If $\Psi$ is a diffeomorphism satisfying either of the above with $\ell=1$, and $f \in \underline{n}_{k}^{1}$, then $\Psi_{*} f \in \underline{n}_{k}$.

Any endomorphism $\Psi$ that satisfies the first three is called homogeneous of degree $\ell$, written $\Psi \in \mathcal{E}_{\ell}^{1}$.

One may proceed further and introduce similar notions of geometric homogeneity for smooth differential forms and tensor fields, compare [32]. It is worthwhile pointing out that what commonly is called linear may be homogeneous of degree zero or one, depending on its geometric nature. E.g., $A x$ in the linear system $\dot{x}=A x+B u$ stands for the linear vector field $f(x)=\sum_{i, j=1}^{n} a_{i j} x_{i} \frac{\partial}{\partial x_{j}} \in \underline{n}_{0}^{1}$ that is a homogeneous of degree zero (with respect to the standard dilation with $r=(1, \ldots 1)$ or $\nu=\sum_{j} \frac{\partial}{\partial x_{j}}$. However, the matrix exponential $e^{A}$ as in the solution $e^{t A} x_{0}$ of the initial value problem $\dot{x}=A x, x(0)=x_{0}$ as a linear map from $\mathbb{R}^{n}$ to $\mathbb{R}^{n}$ is homogeneous of degree one with respect to the standard dilation $e^{A} \in \mathcal{E}_{1}^{1}$.

One again obtains the expected graded and filtered structures and these spaces and their actions [32].

Proposition 4.4: For $m, m^{\prime}, k, k^{\prime}, \ell, \ell^{\prime} \in \mathbb{R}$ true are

$$
\begin{array}{ll}
\text { - } H_{m} H_{m^{\prime}} \subseteq H_{m+m^{\prime}} . & \text { - } \underline{n}_{k} H_{m}^{1} \subseteq H_{m+k} . \\
\text { - }\left[\underline{n}_{k}^{1}, \underline{n}_{k^{\prime}}^{1} \subseteq \underline{n}_{k+k^{\prime}} .\right. & \text { - } H_{m} \circ \mathcal{E}_{\ell} \subseteq H_{m+\ell} . \\
\text { - } \mathcal{E}_{\ell} \circ \mathcal{E}_{\ell^{\prime}} \subseteq \mathcal{E}_{\ell \ell^{\prime}} . & \text { - } \mathcal{E}_{1 * \underline{n}_{k}^{1} \subseteq \underline{n}_{k}^{1} .} .
\end{array}
$$

In particular, vector fields $f_{j} \in \bigoplus_{k<0} \underline{n}_{k}^{\infty}$ of negative homogeneous degree always generate a nilpotent Lie algebra.

We conclude this section with a general theorem that generalizes the linear criterion for asymptotic stability in terms of the sign of the real part of its eigenvalues to homogeneous systems. That classical theorem geometrically says that an $n$ dimensional linear system is asymptotically stable if and only if its restriction to the union of its (generalized) eigenspaces is asymptotically stable, which in the best case is the union of $n$ one-dimensional systems. However, for stabilization purposes in the setting of homogeneous systems it appears more natural to place the generalizations of the eigenspaces rather than place the poles (or eigenvalues).

From an abstract geometric point of view the geometric homogeneity (with respect to a generator $\nu$ of a group $\Phi$ or $\Delta$ of dilations as above) of a dynamical system $\dot{x}=$ $f(x)$ on $\mathbb{R}^{n}$ induces a dynamical system on the quotient space $Q=\left(\mathbb{R}^{n} \backslash\{0\}\right) / \sim$ under the equivalence relation $\sim \subseteq \mathbb{R}^{n} \times \mathbb{R}^{n}$ defined by $x \sim y$ iff there exists $t \in \mathbb{R}$ such that $\Phi_{t}(x)=y$. Analogous to the notion of eigenspaces of a linear system, call the orbits of the action of the dilation group eigenrays. The quotient space $Q$ of all eigenrays may be conveniently identified with any nonzero level set of a smooth Lyapunov function for $-\nu$, which is homomorphic to the standard sphere $S^{n-1} \subseteq \mathbb{R}^{n}$. Write $\pi:\left(\mathbb{R}^{n} \backslash\{0\}\right) \mapsto Q$ for the associated canonical projection. If $f \in \underline{n}_{0}$, i.e., if $\nu$ and $f$ commute, then so do their flows. If $f \in \underline{n}_{k}$ for some $k \neq 0$ then one may rescale time along each nonzero integral curve of $f$, which amounts to multiplying $f$ by a scalar positive homogeneous function $\varphi \in H_{-k}$ (defined on $\left.\mathbb{R}^{n} \backslash\{0\}\right)$ so that the resulting field $\varphi f \in \underline{n}_{0}$ is homogeneous of degree zero. Since such rescaling does not change stability properties, the sequel assumes $f \in \underline{n}_{0}$ throughout.

In this case the flow $\Psi$ of $f$ on $\mathbb{R}^{n} \backslash\{0\}$ directly projects to a continuous flow $\Psi_{\pi}$ on $Q$ (with $Q$ equipped with the quotient topology). Directly following [32], let $\Omega \subseteq Q$ denote the closure of the union of all $w$-limit sets of $\Psi_{\pi}$. As $\Omega$ is invariant under $\Psi_{\pi}$, the associated homogeneous cone $C \Omega=\pi^{-1} \Omega \cup\{0\}$ is invariant under $\Psi$ and hence the dynamical system $\Psi$ restricts to a continuous dynamical system $\Psi_{0}=\left.\Psi\right|_{C \Omega}$. The main result [31], [32] is

Theorem 4.5: If $x=0$ is asymptotically stable for $\Psi_{0}$ then $x=0$ is asymptotically stable for $\Psi$.

This theorem is readily applied for feedback stabilization of homogeneous systems. One uses homogeneous feedback 
$u=k(x)$ such that the induced dynamics $\Psi_{0}$ on $Q$ has a small limit set $\Omega$ such that $x_{0}$ is asymptotically stable for the restricted dynamics $\Psi_{0}$. For many homogeneous examples of three dimensional systems, similar to (14) it is feasible to use feedback to make $\Omega$ a single point (after accounting for another $Z_{2}$ symmetry for odd systems) which is the common $\alpha$ and $\omega$-limit set of all orbits on $Q / \mathbb{Z}_{2}$. This phase diagram is equivalent to that of a linear three dimensional system with a single Jordan block corresponding to a triple eigenvalue. Then the reduced dynamics is one dimensional, and if $\Omega$ can be properly placed, one has achieved asymptotic feedback stabilization, compare [9], [31] for detailed examples.

\section{RECENT AND CURRENT EFFORTS}

The preceding sections focused on how homogeneity properties facilitate studies of controllability, stabilization and related properties for finite dimensional, continuous-time systems. Recent and ongoing work exploits homogeneity properties for both more specific objectives in specialized settings, and for many different classes of systems, especially in infinite dimensions.

For example, much of the above immediately carries over to discrete-time (controlled) dynamical systems, compare, e.g. classic work by [12], [33].

Related notions of stability such as input-to-state-stability (ISS) are studied in, e.g. [34]. Homogeneity is not required for finite-time stabilization, but it much facilitates its analysis and thus has been a popular tool in analysis and design of finite-time stabilizability. This is particular beneficial in systems which exhibit noise and uncertainties in which case sliding-mode control techniques and homogeneity provide desired robustness properties, compare, e.g. [35], [36], and the article [37] in this tutorial session. This latter extends prior results from single-input-single-output (SISO) systems to multiple-input-multiple-output (MIMO) systems.

In a different direction, rather than utilizing homogeneity in the study of other properties and objectives, various recent efforts apply techniques utilizing homogeneity to other classes of dynamical systems. Selected such efforts, still in the context of deterministic systems, focus on extending the techniques to infinite dimensional systems, including the articles [38], [39], [40] in this tutorial session.

Models that incorporate time-delays are especially popular in the bio-medical sciences, and more recently also in dynamical systems that model communication networks. The articles [16], [38] demonstrate how homogeneity can be beneficially utilized in such infinite dimensional dynamical systems.

The article [39] develops a general theoretical framework of geometric notions of homogeneity that apply to evolution equations in any Banach spaces. Careful attention is to be given to the continuity properties of the semigroup of dilations in infinite dimensions. Specific results include scalability and stability properties of solutions. It includes applications to specific nonlinear partial differential equations such the Korteweg-de Vries equation and the SaintVenant equation.
In contrast, the article [40] is an example whose main focus is less on developing general notions of homogeneity and associated tools. Instead it demonstrates how homogeneity comes into play as one of several key ingredients to solve control problems in nonlinear partial differential equations. In this specific example, with applications to reactiondiffusion equations and the Ginzburg-Landau equation, homogeneity properties facilitate the controllability analysis of a nonlinear coupling in a system of parabolic equations.

\section{REFERENCES}

[1] S. P. Bhat and D. S. Bernstein, "Geometric homogeneity with applications to finite-time stability," Math. Control Signals Systems, vol. 17, no. 2, pp. 101-127, 2005. [Online]. Available: http: //dx.doi.org.ezproxy1.lib.asu.edu/10.1007/s00498-005-0151-x

[2] E. Moulay and W. Perruquetti, "Finite time stability and stabilization of a class of continuous systems," J. Math. Anal. Appl., vol. 323, no. 2, pp. 1430-1443, 2006. [Online]. Available: http: //dx.doi.org.ezproxy1.lib.asu.edu/10.1016/j.jmaa.2005.11.046

[3] M. Shub and S. Smale, "Complexity of Bezout's theorem. IV. Probability of success; extensions," SIAM J. Numer. Anal., vol. 33, no. 1, pp. 128-148, 1996. [Online]. Available: http: //dx.doi.org.ezproxy1.lib.asu.edu/10.1137/0733008

[4] H. G. Hermes, "Nilpotent approximations of control systems and distributions," SIAM J. Control Optim., vol. 24, no. 4, pp. 731-736, 1986. [Online]. Available: http://dx.doi.org.ezproxy1.lib.asu.edu/10. $1137 / 0324045$

[5] H. J. Sussmann, "Lie brackets and local controllability: a sufficient condition for scalar-input systems," SIAM J. Control Optim., vol. 21, no. 5, pp. 686-713, 1983.

[6] — "A general theorem on local controllability," SIAM J. Control Optim., vol. 25, no. 1, pp. 158-194, 1987.

[7] W. P. Dayawansa, C. F. Martin, and S. Samelson, "Asymptotic stabilization of a generic class of three-dimensional homogeneous quadratic systems," Systems Control Lett., vol. 24, no. 2, pp. 115-123, 1995. [Online]. Available: http://dx.doi.org.ezproxy1.lib.asu.edu/10. 1016/0167-6911(94)00040-3

[8] M. Kawski, "Stabilization of nonlinear systems in the plane," Systems Control Lett., vol. 12, no. 2, pp. 169-175, 1989. [Online]. Available: http://dx.doi.org.ezproxy1.lib.asu.edu/10.1016/0167-6911(89)90010-8

[9] - "Homogeneous stabilizing feedback laws," Control Theory Adv. Tech., vol. 6, no. 4, pp. 497-516, 1990.

[10] — " "Control variations with an increasing number of switchings," Bull. Amer. Math. Soc. (N.S.), vol. 18, no. 2, pp. 149-152, 1988. [Online]. Available: http://dx.doi.org.ezproxy1.lib.asu.edu/10. 1090/S0273-0979-1988-15630-3

[11] R. Sepulchre and D. Aeyels, "Stabilizability does not imply homogeneous stabilizability for controllable homogeneous systems," SIAM J. Control Optim., vol. 34, no. 5, pp. 1798-1813, 1996. [Online]. Available: http://dx.doi.org.ezproxy1.lib.asu.edu/10.1137/ S0363012994267303

[12] L. Grüne, "Homogeneous state feedback stabilization of homogenous systems," SIAM J. Control Optim., vol. 38, no. 4, pp. 1288-1308 (electronic), 2000. [Online]. Available: http://dx.doi.org.ezproxy1.lib. asu.edu/10.1137/S0363012998349303

[13] C. Qian and W. Lin, "A continuous feedback approach to global strong stabilization of nonlinear systems," IEEE Trans. Automat. Control, vol. 46, no. 7, pp. 1061-1079, 2001. [Online]. Available: http://dx.doi.org.ezproxy1.lib.asu.edu/10.1109/9.935058

[14] J.-M. Coron and L. Praly, "Adding an integrator for the stabilization problem," Systems Control Lett., vol. 17, no. 2, pp. 89-104, 1991. [Online]. Available: http://dx.doi.org.ezproxy1.lib.asu.edu/10. 1016/0167-6911(91)90034-C

[15] R. T. M'Closkey and R. M. Murray, "Exponential stabilization of driftless nonlinear control systems using homogeneous feedback," IEEE Trans. Automat. Control, vol. 42, no. 5, pp. 614-628, 1997. [Online]. Available: http://dx.doi.org.ezproxy1.lib.asu.edu/10.1109/9. 580865

[16] E. Moulay, M. Dambrine, N. Yeganefar, and W. Perruquetti, "Finitetime stability and stabilization of time-delay systems," Systems Control Lett., vol. 57, no. 7, pp. 561-566, 2008. [Online]. Available: http://dx.doi.org.ezproxy1.lib.asu.edu/10.1016/j.sysconle.2007.12.002 
[17] M. A. Krasnosel'skiǔ and P. P. Zabrě̌ko, Geometrical methods of nonlinear analysis, ser. Grundlehren der Mathematischen Wissenschaften [Fundamental Principles of Mathematical Sciences]. Springer-Verlag, Berlin, 1984, vol. 263, translated from the Russian by Christian C. Fenske. [Online]. Available: http://dx.doi.org.ezproxy1.lib.asu.edu/10.1007/978-3-642-69409-7

[18] R. W. Brockett, "Asymptotic stability and feedback stabilization," in Differential geometric control theory (Houghton, Mich., 1982), ser. Progr. Math. Birkhäuser Boston, Boston, MA, 1983, vol. 27, pp. 181-191.

[19] J.-M. Coron, "On the stabilization in finite time of locally controllable systems by means of continuous time-varying feedback law," SIAM J. Control Optim., vol. 33, no. 3, pp. 804-833, 1995. [Online]. Available: http://dx.doi.org.ezproxy1.lib.asu.edu/10.1137/S0363012992240497

[20] F. Ancona, "Normal forms for vector fields with respect to an arbitrary dilation," NoDEA Nonlinear Differential Equations Appl., vol. 3, no. 3, pp. 305-322, 1996. [Online]. Available: http://dx.doi.org.ezproxy1.lib.asu.edu/10.1007/BF01194069

[21] L. Rosier, "Homogeneous Lyapunov function for homogeneous continuous vector fields," Systems Control Lett., vol. 19, no. 6, pp. 467-473, 1992. [Online]. Available: http://dx.doi.org.ezproxy1.lib.asu. edu/10.1016/0167-6911(92)90078-7

[22] H. J. Sussmann, "A product expansion for the Chen series," in Theory and applications of nonlinear control systems (Stockholm, 1985). Amsterdam: North-Holland, 1986, pp. 323-335.

[23] E. Gehrig and M. Kawski, "A Hopf-algebraic formula for compositions of noncommuting flows," in Proceedings IEEE Conference Decision and Control, Cancun, Mexico, 2008, pp. 156-1574.

[24] H. G. Hermes, "Nilpotent and high-order approximations of vector field systems," SIAM Rev., vol. 33, no. 2, pp. 238-264, 1991. [Online]. Available: http://dx.doi.org.ezproxy1.lib.asu.edu/10.1137/1033050

[25] G. Stefani, "On the local controllability of a scalar-input control system," in Theory and applications of nonlinear control systems (Stockholm, 1985). Amsterdam: North-Holland, 1986, pp. 167-179.

[26] M. Kawski, "The combinatorics of nonlinear controllability and noncommuting flows," in Mathematical control theory, Part 1, 2 (Trieste, 2001), ser. ICTP Lect. Notes, VIII. Abdus Salam Int. Cent. Theoret. Phys., Trieste, 2002, pp. 223-311.

[27] R. Goodman, "Approximating Lie algebras of vector fields by nilpotent Lie algebras," in Analyse harmonique sur les groupes de Lie (Sém., Nancy-Strasbourg 1976-1978), II, ser. Lecture Notes in Math. Springer, Berlin, 1979, vol. 739, pp. 293-307.

[28] R. W. Goodman, Nilpotent Lie groups: structure and applications to analysis, ser. Lecture Notes in Mathematics, Vol. 562. SpringerVerlag, Berlin-New York, 1976.

[29] H. Hermes, "Homogeneous feedback controls for homogeneous systems," Systems Control Lett., vol. 24, no. 1, pp. 7-11, 1995. [Online]. Available: http://dx.doi.org.ezproxy1.lib.asu.edu/10. 1016/0167-6911(94)00035-T

[30] - "Homogeneous coordinates and continuous asymptotically stabilizing feedback controls," in Differential equations (Colorado Springs, CO, 1989), ser. Lecture Notes in Pure and Appl. Math. Dekker, New York, 1991, vol. 127, pp. 249-260.

[31] M. Kawski, "Families of dilations and asymptotic stability," in Analysis of controlled dynamical systems (Lyon, 1990), ser. Progr. Systems Control Theory. Birkhäuser Boston, Boston, MA, 1991, vol. 8, pp. 285-294.

[32] - "Geometric homogeneity and applications to stabilization," in Nonlinear Control Systems Design. Elsevier, 1995, pp. 147-152.

[33] H. Hammouri and S. Benamor, "Global stabilization of discrete-time homogeneous systems," Systems Control Lett., vol. 38, no. 1, pp. 5-11, 1999. [Online]. Available: http://dx.doi.org.ezproxy1.lib.asu. edu/10.1016/S0167-6911(99)00040-7

[34] E. Bernuau, A. Polyakov, D. Efimov, and W. Perruquetti, "Verification of ISS, iISS and IOSS properties applying weighted homogeneity," Systems Control Lett., vol. 62, no. 12, pp. 1159-1167, 2013. [Online]. Available: http://dx.doi.org.ezproxy1.lib.asu.edu/10.1016/j. sysconle.2013.09.004

[35] A. Levant, "Universal single-input-single-output (SISO) sliding-mode controllers with finite-time convergence," IEEE Trans. Automat. Control, vol. 46, no. 9, pp. 1447-1451, 2001. [Online]. Available: http://dx.doi.org.ezproxy1.lib.asu.edu/10.1109/9.948475

[36] T. Floquet, J.-P. Barbot, and W. Perruquetti, "Higher-order sliding mode stabilization for a class of nonholonomic perturbed systems," Automatica J. IFAC, vol. 39, no. 6, pp. 1077-1083, 2003.
[Online]. Available: http://dx.doi.org.ezproxy1.lib.asu.edu/10.1016/ S0005-1098(03)00076-1

[37] A. Levant, "Homogeneity of differential inclusions: Application to sliding modes," in Proceedings European Control Conference, Linz, Austria, 2015

[38] D. Efimov, W. Perruquetti, and J.-P. Richard, "Homogeneity concept for time-delay systems," in Proceedings European Control Conference, Linz, Austria, 2015.

[39] A. Polyakov, D. Efimov, E. M. Fridman, and W. Perruquetti, "Homogeneous evolution equation in a banach space," in Proceedings European Control Conference, Linz, Austria, 2015.

[40] J.-M. Coron, S. Guerrero, and L. Rosier, "Homogeneity applied to the controllability of a system of parabolic equations," in Proceedings European Control Conference, Linz, Austria, 2015. 